\title{
De la condición inmunitaria del Urstaat a la construcción de una biopolítica afirmativa
}

\section{From the immunitary natura of the Urstaat to the construction of affirmative biopolitics}

\section{Pamela Soto García, Ricardo Espinoza Lolas}

Pontificia Universidad Católica de Valparaíso, Valparaíso, Chile

\section{Resumen}

Este artículo pretende identificar algunos conceptos de la filosofía de Gilles Deleuze para la construcción de una biopolítica afirmativa en la obra de Roberto Esposito. Para presentar esta propuesta de lectura y análisis es necesario atender al modo como el concepto desterritorialización, opera en el estudio que realiza Esposito de la Italian theory, y así especificar la preocupación epistemológica en la que se inscribe la diferencia italiana como una geofilosofía, que no prentende establecerse como un antecedente para la construcción de un pensamiento de orden nacional y estadocéntrico. El análisis desde el término desterritorialización contribuirá, además, en un segundo apartado a precisar, desde el campo de lo político, la relación entre ambos filósofos, al vincular el Urstaat que describe Gilles Deleuze con las reflexiones acerca del Estado inmunitario que propone Roberto Esposito, la aproximación entre ambos conceptos se realizará a partir de las reflexiones deleuzianas acerca de la "máquina despótica-bárbara", la que entrega

PSG: Doutora em Filosofia, e-mail: pamela.soto@pucv.cl REL: Doutor em Filosofia, e-mail: ricardo.espinoza@pucv.cl 
elementos a través de los que es posible cruzar ambos términos desde el problema de la vida y, de este modo, aproximarse a una reflexión biopolítica. El desarrollo del análisis crítico entre los términos política y vida se abordará en un tercer apartado, a través de la identificación del concepto de lo impersonal, que contribuirán a la elaboración de una biopolítica afirmativa en la obra de Roberto Esposito, además, esta aproximación también apuntará a cuestionar el Estado-nación como organización política privilegiada, proyectando desde esta crítica los alcances epistemológicos y políticos que surgen del cruce entre ambas filosofías.

Palabras clave: Esposito. Deleuze. Desterritorialización. Urstaat. Biopolítica afirmativa.

\section{Abstract}

This article is intended to identify concepts of Gilles Deleuze's philosophy for the construction of affirmative biopolitics in Roberto Esposito's work. In order to present this reading and analysis proposal, it is necessary to address the way the concept of "deterritorialization" works in Esposito's analysis of the Italian theory and to specify the epistemological concern to which the Italian difference belongs as a geophilosophy that does not expect to be established as a precedent for the construction of a national state-centered thinking. The analysis from the concept of "deterritorialization" will also contribute, in a second part, to specify, from the field of politics, the relation between both philosophers by linking the Urstaat described by Gilles Deleuze with the reflections about the immunitary State proposed by Roberto Esposito. The approach between both concepts will be carried out from the Deleuzian reflections about the "barbarian despotic machine", which provides elements through which it is possible to cross both concepts from the problem of life, thus getting closer to a biopolitical reflection. The development of the critical approach analysis between the concepts of "politics" and "life" will be addressed in a third part through the identification of some of those concepts, such as the impersonal, which will contribute to the construction of affirmative biopolitics in Roberto Esposito's work. However, this approach will also aim to questioning the Statenation as a privileged political organization and project this critique to the epistemological and political effects of both philosophies.

Keywords: Esposito. Deleuze. Deterritorialization. Urstaat. Affirmative Biopolitics. 


\section{Introducción: El cruce filosófico entre Gilles Deleuze y Roberto Esposito}

El investigador argentino Marcelo Antonelli publica el año 2013 el artículo "La deriva deleuziana de Roberto Esposito", en este texto establece como hipótesis de lectura que existe "una deriva deleuziana cada vez más marcada en el derrotero filosófico del autor italiano" (2013, p. 39). Sin bien, Antonelli se ocupa de identificar y analizar algunos de los conceptos de la filosofía de Deleuze que utiliza Esposito, no aborda de forma específica las relaciones que se pueden establecer, entre ambos pensamientos, desde la crítica que la filosofia contemporánea realiza al Estado-nación.

El intento por relacionar el pensamiento de ambos autores tiene como antecedente al propio Roberto Esposito, quien considera relevante la filosofía del francés para la construcción de una biopolítica afirmativa: "Deleuze invita a deshacer este nudo biojurídico entre vida y norma de manera que, en vez de separarlas, reconozca la una en la otra, encuentre en la vida su norma inmanente y restituya a la norma el poder en devenir de la vida" (ESPOSITO, 2006 , p. 312). Esta forma de comprender la vida, descentrada de aquellos procesos de subjetivación que apuntan a establecer la condición trascendental de la conciencia como horizonte, se expresan más bien como inmanecia de un territorio en permante proceso de desterritorialización, y es precisamente la condicion de este giro en la concepción de la vida humana aquello que puede fisurar el paradigma inmunitario que se instaura a partir de la modernidad. Esta relación entre las filosofía de Roberto Esposito y Gilles Deleuze, no pretende invisibilizar o desplazar la herencia foucoultiana en la obra del italiano, sino presentar otro antecedente teórico para explorar su obra.

Para dar cuenta de la propuesta epistemológico y política del cruce entre ambas filosofías, se presentará este texto en tres apartados: en el primero de ellos se expondrá la posición de Roberto Esposito al interior de la diferencia italiana - la Italian Theory - , desde la conceptualización epistemológica a la que alude la desterritorialización de Gilles Deleuze, a fin de fisurar desde las implicancias 
de ésta la condición Estado-céntrica y nacionalista que se impone a partir de la modernidad en el pensamiento político. En un segundo momento se abordará el concepto de desterritorialización desde el campo de lo político, vinculando el análisis-crítico de los términos, Urstaat en el pensamiento de Deleuze con el de Estado inmuntario que describe Esposito. En el tercer apartado, a partir de la reflexión del Urstaat como Estado inmunitario se expondrá como Roberto Esposito intenta posicionar, a partir del influjo deleuziano en su obra, algunos elementos para la construcción de una biopolítica afirmativa.

\section{La desterritorialización en el pensamiento de Roberto Esposito}

Roberto Esposito en el libro, Pensiero vivente origine e attualitá della filosofía italiana (2010), expone, a partir de un recorrido por algunas antologías acerca de la filosofía italiana contemporánea, aquello que puede ser considerado como su diferencia. La revisión que realiza Esposito, tiene como antecedente tres libros publicados en inglés, entre los años 1988 y 2009, en los que se expresan las tensiones y las continuidades de la diferencia italiana, denominada como Italian Theory.

El primer texto que revisa es Recording Metaphysics. The New Italian Philosophy (1988), el que se encuentra dirigido por Giovanna Borradori. De acuerdo a lo que señala el propio Esposito (2010) este texto enfatiza el carácter de continuidad del pensamiento italiano “en relación con los grandes problemas de la filosofía europea, otorgando sólo cierto crédito metodológico a su diferencia, en cuanto, se rescata la dimensión historicista que entregan los pensadores a los problemas" (SOTO GARCÍA, 2015, p. 70). El principal mérito de este texto es que inaugura una reflexión acerca de la filosofía contemporánea escrita en Italia, lo que proporciona los primeros antecedentes para dar cuenta de la especificidad de esta producción filosófica. 
El segundo texto Radical Thought in Italy. A Potencial Politicis (1996) estuvo dirigido por Michael Hardt y Paolo Virno. Esposito señala que este texto permite focalizar la diferencia italiana en la reflexión política de su época, la cual tiene como contexto las disputas políticas de los años sesenta y setenta en Italia ${ }^{1}$. A partir de este texto la Italian theory comienza a ser identificada como pensamiento político. Los intelectuales partícipes en este proyecto durante la década de los sesenta y setenta "dividían su trabajo teórico entre la revista Centauro, que responde a una lectura político-teórica, y Laboratorio político, que pretendía dar curso a un proyecto político ligado a la disolución del capitalismo" (SOTO GARCÍA, 2015, p. 72). Roberto Esposito, participa en Il Centauro. Rivista di filosofía e teoría política (ESPOSITO, 2012a), que tiene como propósito interpretar desde la filosofía el quiebre epocal en el que viven, de ahí que la figura del Centauro como expresión de la conjunción de dos elementos diversos en la vida humana es una ilustrativa imagen para la revista.

La tercera antología es la de Lorenzo Chiesa y Alberto Toscano: The Italian Difference between Nihilism and Biopolitics (2009), en la cual se presenta el nihilismo ${ }^{2}$ y la biopolítica como dos de los elementos que diferencian y determinan al pensamiento político italiano contemporáneo, destacando la singularidad de cada uno de ellos, para desde ahí proyectarlo hacia otros ámbitos:

1 Es similar la posición de Katsiaficas al respecto: “En 1977, la combinación de crisis políticas sistemáticas, veloces cambios económicos y una oposición popular creciente culminaron en una revuelta contra el sistema establecido y la oposición comunista más leal. Las subsiguientes acciones guerrilleras de organizaciones como las Brigadas Rojas (BR) ayudaron a nutrir una represión masiva por parte del gobierno y el repliegue de muchas personas de las filas del activismo; pero no sin que antes los movimientos autónomos hubieran transformado el paisaje de la sociedad italiana; las mujeres obtuvieron mayor protección legal y libertad social; los trabajadores elevaron su nivel de vida y aumentaron su tiempo libre; y los jóvenes se liberaron de los residuos del feudalismo patriarcal y de su benévola postergación en las universidades, escuelas y familias." Katsiaficas, G. La subversión de la política. Movimientos sociales autónomos europeos y la descolonización de la vida cotidiana. México DF: UACM, 2013, p. 89.

2 En el año 2000 Esposito, Galli y Vitiello compilan Nichilismo e politica. Texto que permite sustentar la tesis de Chiesa y Toscano, pues precisamente en el prefacio de este texto aquello que se señala es el interés por pensar el vínculo entre ambos términos: "Para prescindir de las diferentes declinaciones a las cuales el fenómeno nihilista es reducido, todas las intervenciones del libro convergen en asumirlo como la custión decisiva de nuestro tiempo - de conformidad con la enigmática intuición de Walter Benjamin, para quien el nihilismo constituye también el'método' de la 'acción política mundial"' (p. 11). 
Es verdad que ambos [términos] nacen originariamente en otro parte - el primero en Alemania y el segundo en Francia - pero aquello no le quita que haya sido la propia elaboración italiana a permitir, o provocar, una difusión siempre más vasta. Esto vale sobretodo para la categoría de biopolítica, por ahora establemente instalada al centro de la discusion, filosófica, política y jurídica internacional (ESPOSITO, 2010, p. 5).

Este itinerario bibliográfico le permite a Esposito delimitar a la Italian theory, como un campo de reflexión filosófica en la que se cruzan nihilismo y biopolítica, y cuyos inicios teóricos se encuentran vinculados a una izquierda italiana que busca renovar el pensamiento filosófico, a partir tanto del rescate de la dimensión histórica en la que la filosofía debiese siempre desenvolverse, como del sustrato en la cual acontece. En este punto es necesario indicar, enfáticamente, que el propio Esposito señala que bajo ningún respecto su trabajo o el de la Italian theory apunta a una filosofía de carácter nacional o territorial, sino a una geofilosofía que da cuenta de "un insieme di caratteristiche ambientali, linguistiche, tonali che rimandano a una modalità specifica e inconfondibile rispetto ad altri stili di pensiero"3 (ESPOSITO, 2010, p. 14), otorgando con ello elementos para pensar en el desarrollo de una filosofía vinculada a la vida.

En este punto el concepto de desterritorialización deleuziano adquiere relevancia en el pensamiento de Esposito, pues la geofilosofía italiana a la que se hace referencia alude directamente al modo como Deleuze, junto a Guattari, definen el proceso de desterritorialización en ¿Qué es filosofía? :

Pensar no es un hilo tensado entre sujeto y un objeto, ni una revolución de uno alrededor de otro. Pensar se hace más bien en relación entre el territorio y la tierra [...] La tierra no es un elemento cualquiera entre los demás, aúna todos los elementos en un mismo vínculo, pero utiliza uno u otro para desterritorializar el territorio (DELEUZE; GUATTARI, 2011, p. 86).

3 La dimensión geofilosófica expresa una determinación sistemática de la diferencia italiana, la que se puede encontrar desarrollada en los textos de Massimo Cacciari. Europa o la filosofía. (2007) y Geofilosofía dell'Europa (1994). 
Esta precisión acerca del concepto desterritorialización permite sostener que el término Italian theory no alude a un identificación de carácter nacionalista de sus exponentes, sino a un gesto de presentar un pensamiento determinado por una época específica, desterritorializadamente. Lo que a su vez Esposito considera como el núcleo epistemológico de la diferencia italiana; "la grande filosofía italiana di Machiavelli, Bruno, Campanella, Galileo y Vico no acompagna, nè segue, la formazione de lo Stato-nazionale, ma la precede di molto" (ESPOSITO, 2010, p. 20), pues las teorizaciones de estos autores se construyen a contrapelo de la formación y legitimación del Estado-Nación, de modo que la desterritorialización en la Italian Theory opera como un intento por desmontar las categorías políticas de la modernidad desde "una tierra que no coincide con la nación y que más bien se constituye, por una larguísima fase en su ausencia" (2013, p. 20).

De este modo la urgencia por una desterritorialización conceptual, de los términos políticos instaurados por la modernidad, es el primer elemento que determinaría a la diferencia italiana, y describiría el trabajo epistemológico que realiza Esposito con su filosofía, en tanto que "todos los conceptos más influyentes de la tradición política - poder, libertad, democracia - ponen de manifiesto un fondo aporético, antinómico, contradictorio" (ESPOSITO, 2009a, p. 11), por ello siempre abierto para la transformación de su sentido.

De los elementos destacados hasta ahora, podemos afirmar que la filosofía de Roberto Esposito, desde su vínculo con la Italian theory, puede ser definida como un intento por impugnar ciertas categorias y supuestos políticos que operan desde la modernidad, y que han capturado sistemáticamente el despliegue de la vida humana, acercándola a un registro tanatopolítico. Es así que el gran desafío para el pensamiento del italiano es elaborar y proponer una biopolítica afirmativa que rompa con el paradigma de orden inmunitario que acompaña a las categorías políticas inauguradas por la modernidad y que no tienen dificultad en "sacrificar el carácter común de dicha vida" (ESPOSITO, 2012b, p. 25). 


\section{Desterritorializacion y biopolítica: del socius al Urstaat inmunitario}

A través del concepto de desterritorialización Gilles Deleuze no sólo apela a un orden de contenido, sino también a un orden epistemológico en el que la historia debe apuntar a "las contingencias y no a la necesidad; cortes y límites, pero no a la continuidad" (DELEUZE; GUATTARI, 2010, p. 146), de modo tal que a partir del uso de este término es posible cuestionar el andamiaje teórico elaborado por el orden que la onto-teología de la metafísica occidental establece.

La desterritorialización desde esta perspectiva puede ser aplicada a los aparatos de Estado como tales, los que se relacionan con determinaciones como las referidas al territorio y la tierra (DELEUZE, 1996), para Deleuze en esto se juega algo más que una posición epistemológica del problema, pues a partir de estos conceptos es posible posicionar la configuración del campo de lo político desde su filosofía. Para revisar el pensamiento político de Gilles Deleuze es necesario remitirse a los textos que el mismo autor considera como su obra más política: El Anti-Edipo. Capitalismo y esquizofrenia (1972) y Mil mesetas. Capitalismo y esquizofrenia (1980).

En el Antiedipo, la máquina territorial es la primera forma en la que se articula el socius o expresión de lo social. Esta máquina territorial "tiene como pieza a los hombres" (DELEUZE; GUATTARI, 2010, p. 147), que los interioriza en un modelo institucional, que forma memoria entre éstos y sus máquinas, y codifica los flujos de deseo. Esta posición permitíra que el filósofo francés, en un primer momento, cuando haga referencia al campo de lo político en el Antiedipo, lo realice a través de la categoria de "máquina territorial primitiva", que desde la tierra "codifica los flujos de producción, medios de producción, productores y consumidores" (2010, p. 147). La tierra, en tanto, depositaria de las especies cultivables, los utensilios para el cultivo y la organización de lo humanos es "la gran estasis inengendrada, el elemento superior a la producción que condiciona la apropiación y la utilización del suelo" (2010, p. 146). Esto permite que la "máquina territorial primitiva" se instituya como 
una forma para dar cuenta de la organización política a partir de la apropiación y utilización del suelo.

La «máquina territorial» es, por tanto, la primera forma de socius, la máquina de la inscripción primitiva, «megamáquina» que cubre un campo social. No se confunde con las máquinas técnicas [que] ya implica un elemento no humano, actuante, transmisor o incluso motor, que prolonga la fuerza del hombre y permite que posea una cierta liberación (2010: 146-7).

La relación que establece Deleuze entre el socius y lo maquínico, le permite mostrar el decurso del socius, que transita entre una "máquina territorial primitiva", una "máquina despótica bárbara", y una "máquina capitalista", evidenciado que las diferencias entre estas máquinas se vincula a las distintas modulaciones que la formación del socius le otorga a la produccion y al deseo, sin constituir por ello una línea de progreso o de evolución entre estos diversos tipos de máquinas.

A partir de la relación no evolutiva o secuencial entre la topología maquínica de la formación del socius, se puede denominar Urstaat, al modelo paradigmático de todo tipo de Estado en el pensamiento de Deleuze. No obstante, es la "máquina despótica-bárbara" la que posibilita que el Urstaat se presente como "una unidad superior trascendente que integra subconjuntos relativamente aislado que funcionan separadamente y que deja subsisitir las viejas inscripciones territoriales" (PAGOTTO, 2014, p. 95). El análisis del Estado despótico adquiere un lugar especial en el pensamiento de Deleuze, en tanto, está máquina permite el surgimiento "del deseo del deseo del déspota" (2014, p. 101), lo que se grafica en que en este tipo de socius no habría meta más alta para la humanidad que la conformación del Estado, y por consiguiente servir a éste.

Estas características del Urstaat vinculado a la "máquina despótica bárbara" puede ser equiparado al concepto de Estado de herencia hobessiana que describe y cuestiona Roberto Esposito. El filósofo italiano discutirá el hecho que a partir de la modernidad, el Estado se ha autoinstituido como el único garante de los derechos de los ciudadanos, aún cuando para ello deban ceder irrevocablemente sus propios 
derechos a "una estructura de dominación supra-individual" (ROJAS CASTRO, 2013, p. 108). Esto implicará que los individuos sólo se transforman en sujetos, si ceden su poder de manera irrevocable e irreversible a esta instancia superior, direccionando unívocamente el proceso de subjetivación.

Con [...] el fin de la seguridad - los hombres sacrifican lo más precioso que tienen, los propios derechos y los propios poderes, a favor de un Tercero, justamente el Estado, capaz de defenderlos y de dar miedo con sus leyes a cualquiera que intente violarlas ${ }^{4}$ (ESPOSITO, 2013, p. 18).

Para Deleuze, el Urstaat no se formó paulatinamente, "sino que surgió ya armado, golpe maestro de una vez" (2010, p. 224). De modo que el socius que se inaugura desde la "máquina despótica-bárbara", puede ser vinculado a lo que Roberto Esposito hace referencia cuando describe el orden potestativo del Estado Moderno, señalando como la construcción de Estado se sostiene en "la formulación del pacto entre sujetos que no obstante deberán fundarlo" (2012b, p. 40). Esta presuposición despoja violentamente al ser humano de todo acerbo político, como si su principal tarea fuese despolitizar al mismo socius que lo constituye. Esta operatoria de la "máquina despotica-bárbara" redunda en la reducción del campo de lo político, a una formalidad técnica de administración y con ello a la neutralización del campo.

Esta reducción de lo político a una condición técnica, es para Esposito, aquello que posibilita que el Estado a partir de la modernidad construya, utilice y perfeccione una serie de dispositivos e instituciones que mantienen alejados a los seres humanos de lo político, neutralizando el conflicto e integrando a partir de esto "una fuerte tendencia inmunitaria - una tendencia autodefensiva y autoprotectora contra las amenazas externas. Ahora, con el transcurrir del tiempo, este elemento inmunitario se vuelve cada vez más fuerte, hasta asumir un carácter autoinmunitario" (2013, p. 28), que se basa en una "economía política y

4 Es verdad que la metáfora del 'cuerpo político' tiene una tradición más lejana, que refiere primer lugar a Platón, pero que con Hobbes, conectada con la metáfora de la máquina, asume un carácter inmunitario - el cuerpo político debe inmunizarse, es decir, protegerse, de los riesgos contenidos en la communitas. (ESPOSITO, 2013, p. 18) 
razón de Estado [...] que emergen desde este magma de transformaciones que están fluyendo al interior de esta nueva forma de racionalidad política que coaccionará y someterá a los individuos, a la vez que producirá sujetos" (ROJAS CASTRO, 2013, p. 110).

Es así que, la "máquina despótica-bárbara" que describe Deleuze, como el Estado moderno inmunitario que ilustra Esposito, dan cuenta de una modificación en la formación del socius, que despoja de todo acerbo político a los seres humanos que lo conforman, neutralizando e inmunizando a partir de ello su acción. Esto desde el "extremo mortífero de la biopolítica" (SALINAS, 2015, p. 283) se denomina como tanatopolítica, y en ella se considera que asegurar la vida consiste en administrar la muerte. Ante esta aparente aporía surge la pregunta, ¿es posible desarticular el carácter inmunitario que acompaña al Urstaat, en tanto expresión de una "máquina despótica bárbara" que administra muerte?

\section{La biopolítica como proceso afirmativo para la vida del socius}

Roberto Esposito inaugura el artículo "Vida biológica y vida política" (2013) con la pregunta “¿Cuál es la relación entre la vida biológica y la vida política o, más simplemente, entre política y vida?” (2013, p. 15). A este cuestionamiento lo sigue una enunciación relevante, que especifica con mayor precisión aquello a lo que alude el filósofo cuando vincula ambos términos.

La política en cuanto organización de las relaciones humanas, siempre ha sido necesaria para la conservación y el desarrollo de la vida. Ninguna sociedad hubiera podido sobrevivir a sus propios conflictos o a los ataques del exterior sin alguna forma de organización política (2013, p. 16).

Ante la estrecha relación que el filósofo establece entre política y vida, podemos precisar la pregunta del apartado anterior señalando:¿De qué modo podemos escapar desde nuestra particular condición humana a una organización política inmunitaria? 
En este punto nuevamente se hace relevante la filosofía de Deleuze para Esposito, debido a que el filósofo francés señala que "la vida escapa a cualquier diagrama de poder, la resistencia al poder descanza en la vida comprendida como fuerza del afuera y se funda en el poder inmanente de la vida misma" (ANTONELLI, 2013, p. 37). Esta condición inmanente de la vida entrega el primer antecedente para la construcción de una biopolítica afirmativa, en cuanto, la vida humana siempre puede romper y escapar a cualquier determinación externa o interna a ella misma - lo que permite que pueda ser denominada como impersonal.

¿Qué quiere decir esto de hablar en nombre propio y no en nombre de otros? No se trata, evidentemente, de que cada cual enfrente a su hora de la verdad, a sus Memorias o a su psicoanálisis, no se trata de la primera persona. Se trata de invocar potencias impersonales, físicas y mentales con las que uno se confronta y contra las que combate desde el momento en que se pretende alcanzar un objetivo del que no se toma conciencia más que en la lucha. En este sentido, el Ser mismo es una cuestión política (DELEUZE, 1996, p. 143).

Este condición impersonal de la vida es a la que alude Esposito cuando hace referencia a una biopolítica afirmativa como la expresión de "una forma de poder sobre la vida sino de poder de la vida - a partir del revés de las prácticas tanatopolíticas" (2013, p. 30), en las que el individuo no se confronta en tanto sujeto solipcista clausurado en un yo, sino desde su condición descentrada o "anorgánica" (LANDAETA; ESPINOZA, 2014, p. 297), es decir, "terceras personas atravesadas y liberadas por el poder de lo impersonal” (ESPOSITO, 2009b, p. 214), porque la "producción de sujetos es inseparable de una desterritorialización que llevan a cabo los propios individuos" (LANDAETA; ESPINOZA, 2014, p. 307).

La posición de lo impersonal le permite a Esposito distinguir en su filosofía entre una política sobre la vida, y una política de la vida, estableciendo con ello un primer elemento para realizar una fisura al carácter inmunitario del Estado Moderno, como representación de la neutralizacion del campo de lo político y despolitización de la 
sociedad, que surge como resultado de la supresión del conflicto como elemento propio de lo político, puesto que una biopolítica afirmativa de lo impersonal, debe romper con todo tipo de dinámica de relación que se constituya a partir de supuestos tanatopolíticos que cercenen la acción humana. Deleuze realiza este ejercició a partir de su crítica al psicoanálisis y a la noción de persona, y son precisamente estos cuestionamientos los que permiten romper con una lectura que el pensamiento occidental parecía clausurar: “Deleuze no sólo individualiza en la ley edípica la estructura general de la exclusión, sino que - cosa aún más relevante para nuestra argumentación- la reduce a un dispositivo jurídico de la persona, tal como fue formulado por el derecho romano" (ESPOSITO, 2009b, p. 207).

En términos deleuzianos podemos afirmar que todo Urstaat, incluso aquel de condición democrática, conserva un vínculo con el despotismo, por lo cual, no existiría un modelo de gobierno mejor a otro, sino prácticas de desterritorialización que permiten siempre cuestionar lo formación de un socius, desde funciones regulatorias que el Estado realiza acerca de si las prácticas de la vida se articulan desde la promoción de una vida plural y creadora, en oposición al individuo como sujeto fruto de una conciencia clausurada sobre la interioridad de sí mima. La subjetivación para Deleuze quedará definida "esencialmente en la invención de nuevas posibilidades vitales" (1996, p. 148), las que variarán según las épocas, y los diversos dispositivos o instituciones que las alberguen, “Tanto es así que [...] el poder no cesa de recuperarlos y de someterlos a las relaciones de fuerzas, y ellos no cesan de renacer y de inventar infinitamente nuevas modalidades"' (1996, p. 160).

De este modo la única forma posible de fisurar el decurso del Urstaat, como Estado inmunitario, es recuperar la condición afirmativa de la vida, condición que se establece descentrando las barreras que la perspectiva de un sujeto solipcista establece al cuestionar "la organización de una normalidad que guía la experiencia del cuerpo, como si se tratase de su propio destino" (LANDAETA; ESPINOZA, 2004, p. 300).

Vivir la vida desde su condición de impersonal, permite desterritorializar la "máquina despótica-bárbara" la que se posicionó a partir del arrebato de los derechos de los seres humanos y de la conciencia 
como territorio privado y clausurado para su intimidad. No obstante, una vida humana que se viva en constante proceso de territorialización y desterritorialización, que increpe - infatigablemente - la organización política del socius al que pertenece, posibilitará que el campo de lo político no se neutralice, ni que su acción personal se despolitice: “Desde el punto de vista de la micropolítica, una sociedad se definen por sus líneas de fuga, que son moleculares. Siempre fluye o huye de algo, que escapa a las organizaciones binarias, al aparato de resonancia, a la máquina de sobrecodificación" (DELEUZE, 2006, p. 220).

Politizar el campo desde los modos de existencia revisando cuáles son las posibilidades para la vida y los procesos de subjetivación es el modo como podemos desterritorializar el Urstaat como Estado inmunitario, que asecha desde la muerte la vida de quienes participan en él, e implementar las bases para la construcción de una biopolítica afirmativa.

\section{Consideraciones finales}

Esta propuesta de lectura nos ha permitido identificar algunos de los conceptos de la filosofía deleuziana presentes en la obra de Roberto Esposito, tales como: desterritorialización, geofilosofía e impersonal, todos ellos con un fuerte impacto en la construcción epistemológica de la filosofía del italiano. La relación, específica entre los términos Urstaat y Estado inmunitario, permitió vincular desde lo político la obra de estos pensadores, al establecer una salida para una biopolítica afirmativa a partir de la concepción de una apertura de los procesos de subjetivación puesta en juego en la obra de Deleuze, lo que permite subvertir cierta clausura presente en el discurso político inmunitario que describe Esposito.

Este itinerario propuesto a través de la filosofía del italiano, a su vez, hizo posible adentrarse a la Italian Theory o diferencia italiana, como una geofilosofía que apuesta por un pensamiento desterritorializado, el que se valora desde su vínculo con contingencia, y cuyo principal impacto se encuentra en la revisión de las categorías políticas 
que a partir de la modernidad han monopolizado la construcción de lo político, y han posicionado al Estado-nación como su único garante.

Esta situación de privilegio que la historia de occidente ha otorgado al Estado, ha permitido establecer al Urstaat como ejemplo paradigmático del ejercicio de un Estado inmunitario, que a través de su acción despótica neutraliza la inmanencia del conflicto que acompaña toda organización política. Este ejercicio de despotenciación o neutralización del conflicto en los procesos de subjetivación humana, que se realiza por medio de la cesión de derechos al Estado-Nación, tiene como efecto una relación tanatológica entre política y vida, transformándose el campo de lo político, en una escenario para la muerte.

Revertir esta operatoria es lo que persigue la biopolítica afirmativa, que a contrapelo de la "máquina despótica bárbara" politiza las relaciones en el socius, de modo tal que permitiría transitar desde la reducción de la democracia entendida como un tipo de modelo de gobierno, hacia el ejercicio de la democracia como potencia afirmativa de la vida, que se resiste a toda neutralización o inmunización desde la diferenciación que implica la vida como emancipación desde la multiplicidad de lo impersonal.

\section{Referencias}

ANTONELLI, M. La deriva deleuziana de Roberto Esposito. Revista Pleyade, n. 12, p. 35-56, jul/dic. 2013.

CACCIARI, M. Europa o la filosofía. Madrid: Antonio Machado Libros, 2007.

DELEUZE, G. Conversaciones, 1972-1990. Valencia: Pre-texto, 1996.

DELEUZE G.; GUATTARI, F. Mil mesetas. Capitalismo y esquizofrenia. Valencia: Pre-texto, 2006.

DELEUZE, G.; GUATTARI, F. El Antiedipo. Capitalismo y esquizofrenia. Buenos Aires: Paidós, 2010.

DELEUZE, G.; GUATTARI, F. ¿Qué es filosofía? Barcelona: Anagrama, 2011.

ESPOSITO, R. Bíos. Biopolítica y filosofía. Buenos Aires: Amorrortu, 2006. 
ESPOSITO, R. Comunidad, inmunidad y biopolítca. Barcelona: Herder, 2009a.

ESPOSITO, R. Tercera persona. Política de la vida y filosofía de lo impersonal. Buenos Aires: Amorrortu, 2009b.

ESPOSITO, R. Pensiero vivente. Orige e attualitá della filosofia italiana. Torino: Einaudi, 2010.

ESPOSITO, R. Comunità e biopolítica. Milano: Mímesis, 2012a.

ESPOSITO, R. Diez pensamiento acerca de la política. Buenos Aires: Fondo de Cultura Económica, 2012b.

ESPOSITO, R. Vida biológica y vida política. Revista Pleyade, n. 12, pp. 15-33, jul/ dic. 2013.

ESPOSITO, R.; GALLI. C; VITIELLO, V. Nihilismo y política. Buenos Aires: Manantial, 2000.

KATSIAFICAS, George. La subversión de la política. Movimientos sociales autónomos europeos y la descolonización de la vida cotidiana. México D. F.: UACM, 2013. LANDAETA MARDONES, P; ESPINOZA LOLAS, R. Geofilosofía de la ciudad para pensar más allá del organismo. Revista Autora, v. 26, n. 38, p. 295-313, jan/ jun. 2014.

PAGOTTO, M.A. Conceptualizaciones sobre el Estado en el Anti-Edipo, capitalismo y esquizofrenia. Revista Eidos, n. 21, p. 85-103, 2014.

ROJAS CASTRO, B. Neoliberalismo y dictadura: el conflicto entre ciudadanía y totalitarismo económico. Revista La Cañada, n. 4, p. 105-135, 2013.

SALINAS ARAYA, ADÁN. La semántica biopolítica. Foucault y sus recepciones. Viña del Mar: Cenaltes, 2015.

SOTO GARCÍA, P. Conflicto y vida: la recuperación del Centauro en el pensamiento de Roberto Esposito. Revista de la Academia, v. 20, p. 67-84, 2015.

Recibido: 10/20/2016

Recebido: 20/10/2016

Aprobado: 02/18/2017

Aprovado: 18/02/2017 